\title{
ARDIŞIK TORAKS VE ABDOMEN BT TETKIKLERINDE TARAMA UZUNLUĞU VE RADYASYON DOZ PARAMETRELERININ KARŞILAŞTIRILMASI
}

\author{
COMPARISON OF SCAN LENGTH AND RADIATION DOSE PARAMETERS \\ IN CONSECUTIVE THORACIC AND ABDOMINAL CT EXAMINATIONS
}

\author{
Eray ATLI', Abidin KILINÇER², Sadık Ahmet UYANIK', Umut ÖĞÜŞLÜ', Halime ÇEVIK CENKERi' \\ 'İstanbul Okan Universitesi Tıp Fakültesi, Radyoloji Bölümü \\ ${ }^{2}$ Selçuk Üniversitesi Tıp Fakültesi, Radyoloji Bölümü
}

öz

\begin{abstract}
AMAÇ: Bu çalışma ile amacımız ardışık toraks ve abdomen BT tetkiklerinde tarama uzunluğu değişkenliğini ve tarama uzunluğunun radyasyon dozu parametreleri üzerine etkisini değerlendirmektir.

GEREÇ VE YÖNTEM: Merkezimizde, Ocak 2018 ve Aralık 2018 tarihleri arasında aynı hastaya ait ardışık toraks $(n=85)$ ve abdomen BT $(n=57)$ tetkikleri çalışmaya dahil edildi. Toraks BT tetkiklerinin \% $39(n=33)^{\prime} u$, abdomen BT tetkiklerinin \% $51(n=29)^{\prime} i$ kadındı. BT radyasyon dozu parametreleri görüntü arşivleme iletişim sisteminden (picture archiving communications system, PACS) retrospektif elde edildi. Hacimsel BT doz indeksi (Volume CT dose index, CTDlvol) ve doz uzunluk çarpımı (dose length product, DLP) değerleri hasta protokolünden kaydedildi. Etkin doz (ED) ve tarama uzunluğu (TU) hesaplandı. Ardışık toraks ve abdomen BT tetkikleri kendi içinde (ilk tetkik ve ikinci tetkik olmak üzere) iki gruba ayrıldı, ve BT radyasyon dozu parametreleri ile TU değerlendirildi.
\end{abstract}

BULGULAR: Toraks ve abdomen BT'si elde edilen hastaların ortalama yaşı sırasıyla $58 \pm 16$ ve $51 \pm 16$ 'dır. Her iki tetkik bölgesinde ardışık BT tetkikleri arasında DLP ve ED değerleri arasında istatiksel fark saptanmadı $(p>0,05)$. Ardışık tetkikler arasında CTDIvol değeri açısından toraks BT grubunda anlamlı fark bulunmazken $(p=0,724)$, abdomen BT grubunda anlamlı fark göstermektedir $(P=0,042)$. Tarama uzunluğunun tetkikler arasındaki ortalama farkı toraks BT için 3,3 $\pm 2,6 \mathrm{~cm}$, abdomen BT için $3,1 \pm 2,5 \mathrm{~cm}$ olarak hesaplandı. Her iki tetkik bölgesinde tarama uzunluğu ardışık tetkikler arasında anlamlı farklılık göstermemektedir $(p>0,05)$.

SONUÇ: Ardışık toraks ve abdomen BT tetkiklerinde, tarama uzunluğu açısından DLP ve ED'ye etki edecek bir farklılık saptanmadı. Ardışık tetkiklerde TU açısından fark saptanmamasına rağmen, $\mathrm{BT}$ çekimlerinde TU taranan anatomik bölgede tanısal bilgi kaybına neden olmayacak en kısa şekilde ayarlanmalıdır.

ANAHTAR KELIMELER: Bilgisayarlı tomografi, Tarama uzunluğu, Hacimsel bilgisayarlı tomografi doz indeksi, Doz uzunluk çarpımı, Etkin doz.

Geliş Tarihi / Received: 23.05.2020

Kabul Tarihi / Accepted: 28.09 .2020

Yazışma Adresi / Correspondence: Dr.Öğr.Üyesi ERAY ATLI

İstanbul Okan Universitesi Tıp Fakültesi, Radyoloji Bölümü

E-mail: atlieray@gmail.com

Orcid No (Sırasıyla): 0000-0002-2639-9924, 0000-0001-6027-874X, 0000-0003-0622-2985, 0000-0001-7985-0734, 0000-0003-1622-7

\section{ABSTRACT}

OBJECTIVE: The aim of this study is to evaluate the scan length variability and the effect of the scan length on radiation dose parameters in consecutive thoracic and abdominal CT examinations.

MATERIAL AND METHODS: Patients who underwent consecutive thoracic $(n=85)$ and abdominal CT $(n=57)$ examinations between January 2018 and December 2018 were included in this study. Thirty-nine percent $(n=33)$ of the thoracic CT examinations and $51 \%(n=29)$ of the abdominal CT examinations were performed on women. CT radiation dose parameters were obtained retrospectively from picture archiving communications system. Volume CT dose index (CTDlvol) and dose length product (DLP) values were recorded from the patient protocols. Effective dose (ED) and scan length (SL) were calculated. Consecutive thoracic and abdominal CT examinations were divided into two groups (first examination and second examination), and CT radiation dose parameters and SL were evaluated.

RESULTS: The mean age of patients in both thoracic and abdominal CT groups were $58 \pm 16$ and $51 \pm 16$ years old, respectively. There was no statistical difference between consecutive CT examinations in the relevant regions in terms of DLP and ED values $(p>0.05)$. CTDlvol were similar between consecutive examinations in the thoracic CT group $(p=0.724)$, whereas there was significant difference between consecutive examinations in the abdominal CT group in terms of CTDIvol $(p=0.042)$. The mean differences of SL in CT examinations were $3.3 \pm 2.6 \mathrm{~cm}$ for thoracic CT group and $3.1 \pm 2.5 \mathrm{~cm}$ for abdominal CT group. There was no significant difference in both consecutive thoracic and abdominal CT examinations in terms of SL ( $p>0.05$ ).

CONCLUSIONS: In consecutive thoracic and abdominal CT examinations, there was no difference in terms of SL that can affect DLP and ED. Although there is no difference in terms of SL between consecutive thoracic and abdominal CT examinations, SL must be adjusted as short as possible since it will not cause any loss in the diagnostic information in the region of interest.

KEYWORDS: Computed tomography, Scan length, Volume computed tomography dose index, Dose length product, Effective dose 


\section{Giriş}

Bilgisayarlı tomografi (BT), X ışını ile görüntü oluşturan bir modalite olup, acil ya da acil dışı hastalıkların tanı ve takibinde kullanılmaktadır.

Türkiye'de 2008 yılında 5.52 milyon olan BT sayısı 2015 yılında 13.68 milyona ulaşmıştır (1).

Yıllar içinde BT'nin artan kullanımı, X-ışını maruziyetine bağlı kanser gelişimi endişesini de artırmaktadır $(2,3)$. Amerika birleşik devletlerinde (ABD), BT tüm görüntüleme yöntemlerinin $\% 12$ sini oluşturmasına rağmen, populasyonda medikal görüntüleme kaynaklı radyasyon maruziyetinin \%50'sini oluşturmaktadır (4). Bilgisayarlı tomografinin yararlarına karşın, kullanımı sınırlandıran başlıca neden X ışınına bağlı radyasyon maruziyetidir. Bu nedenle, öncelikle BT'nin gereksiz kullanımından kaçınılmalı ve hastalar BT kaynaklı radyasyon maruziyetinin zararlı etkilerinden korunmalıdır (5 - 7). BT tetkikleri mümkün olan en düşük radyasyon dozunda ve kabul edilebilir tanısal kalitede elde edilmelidir. BT tetkiklerinde radyasyon dozunu azaltmak için tüp potansiyeli $(\mathrm{kV})$ ve akımı $(\mathrm{mA})$ modülasyonu, gürültü azaltma yöntemleri gibi çeşitli teknikler bildirilmiştir (8 - 10). Doz azaltma yöntemlerinden bir diğeri de oldukça basit bir yöntem olan doğru tarama uzunluğunun ilgili tarama bölümüne göre, ne eksik ne de fazla olacak şekilde, ayarlanmasıdır. Badawy ve ark. (11), BT'de doğru tarama uzunluğu ile her bir taramanın etkin dozu (effective dose, ED) potansiyel olarak 2 mSv'ye kadar azaltılabileceğini bildirmiştir. Bu çalışma ile amacımız ardışık toraks ve abdomen BT tetkiklerinde tarama uzunluğu değişkenliğini ve tarama uzunluğunun radyasyon dozu parametreleri üzerine etkisini değerlendirmektir.

\section{GEREÇ VE YÖNTEM}

\section{Hasta Verilerinin Toplanması}

Merkezimizde, Ocak 2018 ve Aralık 2018 tarihleri arasında aynı hastaya ait ardışık toraks $(n=85)$ ve abdomen $B T(n=57)$ tetkikleri çalışmaya dahil edildi. BT radyasyon dozu parametreleri görüntü arşivleme iletişim sisteminden (picture archiving communications system, PACS) retrospektif elde edildi. Hacimsel BT doz indeksi (Volume CT dose index, CTDlvol) ve doz uzunluk çarpımı (dose length product, DLP) değerleri hasta protokolünden kaydedildi. ED, tetkik DLP değerinin ilgili anatomik bölgenin k katsayısı ile çarpılmasıyla hesaplandı (12). Tarama uzunluğu (TU), DLP'nin CTDlvol değerine bölünmesi ile hesaplandı. Ardışık toraks ve abdomen BT tetkikleri kendi içinde (ilk tetkik ve ikinci tetkik olmak üzere) iki gruba ayrıldı.

\section{Toraks ve Abdomen Bt Tetkiklerinin Çekim Paramet- releri}

Tetkikler 64 kesitli BT'de (Optima CT 660, General Electric Medical Systems, Milwaukee, Wisconsin, $A B D$ ) elde edildi. Toraks ve abdomen BT tetkiklerinin çekim parametreleri verildi (Tablo 1).

Tablo 1: Toraks ve abdomen BT tetkiklerinin çekim parametreleri

\begin{tabular}{|c|c|c|}
\hline Parametreler & $\begin{array}{c}\text { Toraks BT } \\
\mathrm{n}=85\end{array}$ & $\begin{array}{c}\text { Abdomen BT } \\
\quad \mathrm{n}=57\end{array}$ \\
\hline Kesit kalınlı̆̆ı (mm) & 2,5 & 1,25 \\
\hline $\begin{array}{l}\text { Dedektör kapsama } \\
\text { mesafesi }(\mathrm{mm})\end{array}$ & 40 & 40 \\
\hline $\begin{array}{l}\text { Tüp akımı (reference } \\
\text { noise index) (mAs)* }\end{array}$ & $90-400(15,86)$ & $80-450(15,86)$ \\
\hline Tüp potansiyeli $(k V)$ & 120 & 120 \\
\hline $\begin{array}{l}\text { Gantry rotasyon zamant } \\
\text { (s) }\end{array}$ & 0,5 & 0,5 \\
\hline
\end{tabular}

\section{ETIK KURUL}

İstanbul Okan Üniversitesi Etik Kurulundan retrospektif olarak alındı (2019/18) ve hasta onamından feragat edilmiştir.

\section{ISTATIKSEL ANALIZ}

Kalitatif değişkenler sayı ile ifade edildi. Kantitatif değişkenlerin normal dağılım açısından değerlendirilmesi Kolmogorov-Smirnov testi ile yapıldı. Normal dağılmayan değişkenler için ortanca (min-maks) değerleri, normal dağılan değişkenler için ortalama \pm SS verildi. Bağımlı veri olan kantitatif değişkenlerin ilk ve ikinci tetkik grupları arasında karşılaştırılması eşleştirilmiş örneklem T testi veya Wilcoxon testi ile yapıldı. $P$ değeri $0,05^{\prime}$ in altında ise fark istatistiksel anlamlı kabul edildi. Analizler için SPSS v23.0 (IBM, Armonk, New York, Amerika) programı kullanıldi.

\section{BULGULAR}

Toraks BT tetkiklerinin \% $39(n=33)$ 'u, abdomen BT tetkiklerinin \% 51 ( $n=29)$ 'i kadındı. Toraks ve abdomen BT'si elde edilen hastaların ortalama yaşı sırasıyla $58 \pm 16$ ve $51 \pm 16$ 'dır. Ortanca CTDlvol, DLP, ED ve ortalama TU değerleri verildi (Tablo 2). 
Tablo 2: Ardışık toraks ve abdomen BT tetkiklerinin radyasyon dozu parametreleri ile tarama uzunluğunun gruplara göre dağılımı ve $p$ değerleri

\begin{tabular}{|c|c|c|c|c|}
\hline \multicolumn{2}{|c|}{ Parametre } & Illk tetkik & Íkinci tetkik & $\mathbf{p}$ \\
\hline \multicolumn{5}{|c|}{ Toraks BT grubu } \\
\hline CTDI $_{\text {vol }}$ (mGy) & $\begin{array}{l}\text { Ortanca (min- } \\
\text { maks) }\end{array}$ & $7,81(3,26-21,45)$ & $7,96(3,06-16,9)$ & 0,724 \\
\hline DLP (mGy.cm) & $\begin{array}{l}\text { Ortanca (min- } \\
\text { maks) }\end{array}$ & $290(114,2-875,3)$ & $296,5(95,4-647,8)$ & 0,96 \\
\hline ED (mSv) & $\begin{array}{l}\text { Ortanca (min- } \\
\text { maks) }\end{array}$ & $4,06(1,6-12,25)$ & $4,15(1,34-9,07)$ & 0,94 \\
\hline TU (cm) & Ortalama $\pm \mathrm{SS}$ & $38 \pm 3,77$ & $38 \pm 3,94$ & 0,87 \\
\hline \multicolumn{5}{|c|}{ Abdomen BT grubu } \\
\hline CTDI vol $_{\text {(mGy) }}$ & $\begin{array}{l}\text { Ortanca (min- } \\
\text { maks) }\end{array}$ & $8,6(3,44-22,03)$ & $8,64(3,8-22,48)$ & 0,042 \\
\hline DLP (mGy,cm) & $\begin{array}{l}\text { Ortanca (min- } \\
\text { maks) }\end{array}$ & $495(166,8-1428,1)$ & $456(142,3-1276,3)$ & 0,084 \\
\hline ED (mSv) & $\begin{array}{l}\text { Ortanca (min- } \\
\text { maks) }\end{array}$ & $7,42(2,5-21,42)$ & $6,84(2,14-19,15)$ & 0,084 \\
\hline TU (cm) & Ortalama \pm SS & $54 \pm 5,72$ & $53 \pm 6,18$ & 0,29 \\
\hline
\end{tabular}

Her iki tetkik bölgesinde ardışık BT tetkikleri arasında DLP ve ED değerleri arasında istatiksel fark saptanmadı $(p>0,05)$. CTDlvol değeri toraks BT grubunda anlamlı fark göstermezken, abdomen BT grubunda ardışık tetkikler arasında anlamlı fark bulundu ( $P=0,042)$. Tarama uzunluğunun tetkikler arasındaki ortalama farkı toraks BT için 3,3 $\pm 2,6 \mathrm{~cm}$, abdomen BT için 3,1 $\pm 2,5$ $\mathrm{cm}$ olarak hesaplandı. Her iki tetkik bölgesinde tarama uzunluğu ardışık tetkikler arasında anlamlı farklılık göstermemektedir ( $p>0,05)$.

\section{TARTIŞMA}

Bu çalışma ile amacımız ardışık toraks ve abdomen BT tetkiklerinde tarama uzunluğu açısından farklılık varlığı ve tarama uzunluğu değişkenliği değerlendirmektir.

Merkezimizde tarama alanı toraks BT'lerde supraklaviküler fossa ve böbreklerin orta kesimleri arasında iken, abdomen BT'lerde akciğer bazalleri ile inguinal bölge arasındadır. Çalışmamızda ortalama TU değeri toraks BT'lerde ilk ve ikinci tetkik grubunda $38 \mathrm{~cm}$, abdomen BT'lerde ilk tetkik grubunda $54 \mathrm{~cm}$ ve ikinci tetkik grubunda 53 cm'dir. Tarama uzunluğunun aynı hastanın ardışık tetkikleri arasındaki ortalama farkı toraks BT için 3,3 cm, abdomen BT için $3,1 \mathrm{~cm}$ olarak hesaplandı. Bununla beraber her iki tetkik bölgesinde tarama uzunluğu ardışık tetkikler arasında anlamlı farklılık göstermemektedir.

BT tetkiklerinde amaç, öncelikle tanısal kalitede mümkün olan en düşük doz parametreleri ile görüntüleme yapılmasıdır. Bu prensip "as low as reasonably achievable" (ALARA) olarak bilinir.

Bir BT tetkikine ait radyasyon dozu parametreleri CTDlvol ve DLP olup, BT cihazı tarafından has- ta protokolünde verilir. Üçüncü bir radyasyon dozu parametresi olan etkin doz (effective dose, ED) ise radyasyonun biyolojik etkilerini yansitır ve hesaplanır. CTDlvol, DLP ve ED'nin birimleri sırasıyla miligray (mGy), miligray.cm (mGy.cm), and miliSievert (mSv)'tir. Bu üç parametre ile BT tekiklerinin radyasyon dozu karşılaştırma ve yorum yapmak için kullanılabilir.

CTDlvol, başlıca BT cihazı tarafından hastaya verilen radyasyonun yoğunluğunu gösterir ve hastanın ne kadar radyasyona maruz kaldığı anlamına gelmez. Referans olarak alınan 16 veya $32 \mathrm{~cm}$ 'lik bir silindirik fantomda ortalama kesit başına radyasyona maruziyetini ifade eder.

CTDIvol, hasta boyutları ile ilgili değildir, DLP ise CTDlvol ve tarama uzunluğunun çarpımıdır. Bu nedenle DLP, BT tetkiklerinde verilen toplam radyasyon miktarını ifade eder. ED bir diğer radyasyon dozu parametresi olup, tüm organların maruz kaldığı eşdeğer dozu ifade eder. Basitçe ED radyasyonun biyolojik etkilerini yansıtır ve maruz kalınan radyasyon dozlarını karşılaştırılabilir bir forma sokar. BT'nin radyasyon dozunun diğer radyolojik tetkikler, doğal arka plan maruziyetleri (natural background exposures) ile karşılaştırılmasını sağlar. Örneğin bir arka ön akciğer radyografisinin ED değeri 0,05 mSv iken, bir toraks BT tetkikinin ED değerinin $5 \mathrm{mSv}$ olması (13). ED, şu denklemle hesaplanır: $E=k \times$ DLP. İlgili denklemde $k$ faktörü BT tetkiklerinde taranan ilgili anatomik bölge için spesifik bir katsayıdır. 120 kV'de elde edilmiş BT taramaları için bu yöntem ile ED tahmini altın standart organ dozu bazlı tekniğe göre, \% 15 değişkenlik gösterdiği bildirilmiştir (14).

Badawy ve ark. (11) çalışmasında tarama uzunluğunda $10 \mathrm{~cm}$ 'lik bir artış, ED değerinde beyin BT'de \%250, lomber BT'de \% 46, toraks ve abdomen BT tetkiklerinde \%15 oranında artışa neden olmuş. Badawy ve ark. (11) çalışmasında TU toraks BT'lerde 34,1 cm iken, abdomen BT'lerde 50,1 cm'dir. Sing ve ark. (15) ise median TU toraks BT'lerde $33 \mathrm{~cm}$ iken, abdomen BT'lerde $46 \mathrm{~cm}$ olarak bildirilmiştir. Kanal ve ark. (16) tarafından yapılan başka çalışmada ise median TU toraks BT'lerde $35 \mathrm{~cm}$, abdomen BT'lerde $50 \mathrm{~cm}$ 'dir. Çalışmamızın toraks ve abdomen BT tetkiklerinin mean TU değerleri literatür verilerine göre daha yüksektir, toraks ve abdomen 
BT tetkiklerimizde daha kısa TU değeri ile dozda azalma mümkündür. Ortanca CTDlvol ve DLP değerleri ilk ve ikinci tetkik gruplarında toraks BT'lerde yaklaşık 8 mGy ve 300 mGy.cm; abdomen BT'lerde 8,6 mGy ve yaklaşık $500 \mathrm{mGy}$. $\mathrm{cm}$ 'dir. Çalışmamızdaki toraks ve abdomen BT tetkiklerinin ortalama TU değerlerinin literatüre göre yüksek olmasına rağmen, bu değerler Kanal ve ark. (16) 'nın çalışması ile karşılaştırıldığında daha düşüktür (ortanca CTDlvol ve DLP değerleri toraks BT'lerde $10 \mathrm{mGy}$ ve $347 \mathrm{mGy}$. $\mathrm{cm}$; abdomen BT'lerde $13 \mathrm{mGy}$ ve $615 \mathrm{mGy} . \mathrm{cm})$. Ardışık tetkikler arasında CTDlvol değeri açısından toraks BT grubunda anlamlı fark bulunmazken, abdomen BT grubunda anlamlı fark bulundu. Ancak ortanca CTDlvol değeri ilk ve ikinci tetkik grubunda benzerdir (8,6 \& 8,64 mGy). Ayrıca her iki grup arasında DLP ve ED açısından istatistiksel olarak anlamlı fark saptanmadı.

Çalışmamızda iki limitasyon vardır. Birincisi, çalışmanın tek merkezli olması ve elde edilen toraks ve abdomen $\mathrm{BT}$ tetkiklerinin tek $\mathrm{BT}$ cihazı ile elde edilmesidir. İkincisi, toraks ve abdomen BT tetkiklerinin çekimi toplam sayısı 13 olan ve sürekli rotasyon halinde olan radyoloji teknikerleri tarafından yapılmaktadır. Bu nedenle, her ne kadar da ilgili tetkiklerde tarama alanı belli olmasına rağmen, tarama uzunluğunu belirlemede kişiden kişiye farklılık kaçınılmazdır.

\section{SONUÇ}

Çalışmamızda toraks ve abdomen BT tetkiklerinin TU değerleri literatüre göre daha yüksektir, DLP ile ED değerine artışa neden olmaktadır. Ardışık toraks ve abdomen BT tetkiklerinde, tarama uzunluğu açısından DLP ve ED'ye etki edecek bir farklılık saptanmadı. Ardışık tetkiklerde TU açısından fark saptanmamasına rağmen, BT çekimlerinde TU taranan anatomik bölgede tanısal bilgi kaybına neden olmayacak en kısa şekilde ayarlanmalıdır.

\section{KAYNAKLAR}

1. Schmid D. Computed tomography (CT) scan examinations in Turkey 2008-2015. 2018; Available from: https:// www.statista.com/statistics/862506/computed-tomography-scan-examinations-in-turkey/. Erişim 18.05.2020.

2. Berrington de Gonzalez A, Darby S. Risk of cancer from diagnostic X-rays: estimates for the UK and 14 other countries. Lancet 2004;363:345-51.
3. Pearce MS, Salotti JA, Little MP, et al. Radiation exposure from CT scans in childhood and subsequent risk of leukaemia and brain tumours: a retrospective cohort study. Lancet 2012;380:499-505.

4. Schauer DA, Linton OW. NCRP Report No. 160, Ionizing Radiation Exposure of the Population of the United States, medical exposure--are we doing less with more, and is there a role for health physicists? Health Phys 2009;97:1-5.

5. Schauer DA, Linton OW. National Council on Radiation Protection and Measurements report shows substantial medical exposure increase. Radiology 2009;253:293-6.

6. IAEA. International basic safety standards for protection against ionizing radiation and for the safety of radiation sources. International Atomic Energy Agency. 1996 (Safety Series No:115).

7. Santos J, Foley S, Paulo G, McEntee MF, Rainford L. The establishment of computed tomography diagnostic reference levels in Portugal. Radiat Prot Dosimetry 2014;158:307-17.

8. Kalra MK, Maher MM, Toth TL, et al. Strategies for CT radiation dose optimization. Radiology 2004;230:619-28.

9. McCollough $\mathrm{CH}$, Bruesewitz MR, Kofler JM, Jr. CT dose reduction and dose management tools: overview of available options. Radiographics 2006;26:503-12.

10. Strauss KJ, Goske MJ, Kaste SC, et al. Image gently: Ten steps you can take to optimize image quality and lower CT dose for pediatric patients. AJR Am J Roentgenol 2010;194:868-73.

11. Badawy MK, Galea M, Mong KS, U P. Computed tomography overexposure as a consequence of extended scan length. J Med Imaging Radiat Oncol 2015;59:586-9.

12. Christner JA, Kofler JM, McCollough CH. Estimating effective dose for CT using dose-length product compared with using organ doses: consequences of adopting International Commission on Radiological Protection publication 103 or dual-energy scanning. AJR Am J Roentgenol 2010;194:881-9.

13. Huda W, Mettler FA. Volume CT dose index and dose-length product displayed during $\mathrm{CT}$ : what good are they? Radiology 2011;258:236-42.

14. McCollough $\mathrm{CH}$. Patient dose in cardiac computed tomography. Herz 2003;28:1-6.

15. Singh R, Szczykutowicz TP, Homayounieh F, et al. Radiation Dose for Multiregion CT Protocols: Challenges and Limitations. AJR Am J Roentgenol 2019;213:1100-6.

16. Kanal KM, Butler PF, Sengupta D, Bhargavan-Chatfield M, Coombs LP, Morin RL. U.S. Diagnostic Reference Levels and Achievable Doses for 10 Adult CT Examinations. Radiology 2017;284:120-33. 